\title{
Visuelle Stimuli in der qualitativen Forschung
}

\author{
Potenziale und Grenzen des fotogestützten Interviews
}

\section{Von Oliver Dimbath}

Zusammenfassung: Bei vielen Spielarten qualitativ-interpretativer Forschung steht die rekonstruktive Arbeit mit Datenkorpi im Mittelpunkt, die aus erinnerungsfundierten Erzählsequenzen bestehen. Von großer Bedeutung ist, welcher Stimulus gesetzt wird, um die forschungsrelevanten Informationen zu evozieren. Visuelle Medien führten in dieser Funktion über lange Zeit ein Schattendasein. Der Beitrag nimmt die Form des fotogestützten Interviews auf und diskutiert die Methode der fotoelizitativen Befragung als einen Zugang für die Reflexion vergangener Erlebnissequenzen im Interview. Damit wird eine alternative Befragungsform für die qualitativ-rekonstruktive Forschung vorgestellt, bei der dem Problem des Erinnerns ein besonderer Stellenwert zukommt.

\section{Einleitung}

Die Entwicklung neuer Methoden in der Sozialforschung ist nicht allein auf die Innovativität der Forschenden zurückzuführen. Sie erwächst vor allem auch den sozio-kulturellen Rahmenbedingungen, unter denen Forschung stattfindet. Dieser wissenschaftssoziologische Gemeinplatz leitet sich nicht aus der Erkenntnis ab, dass wissenschaftliches Wissen kaum mit Blick auf eine eines Tages zu erreichende ,Wahrheit' oder, Allwissenheit' akkumulativ angehäuft werden kann, sondern aus der Diagnose der Konjunkturzyklen sogenannter Leitdisziplinen oder dem revolutionären Wechsel von Forschungsparadigmen. In den Geistes- und Sozialwissenschaften weist die Kategorie des turns auf eine Wandlungsdynamik hin, die nicht disziplinimmanent, sondern mit Blick auf umfassende Akzentverschiebungen ganzer Wissenschaftsbereiche erklärt werden kann, welche in Wechselwirkung mit dem Denkstil ihrer Zeit zu stehen scheinen.

Im folgenden Beitrag geht es um die Diskussion einer sozialwissenschaftlichen Forschungsmethode, die nicht neu ist, aber durch die kulturelle und insbesondere die technologische Entwicklung einen Relevanzzuwachs verzeichnen dürfte: das fotogestützte Interview. Im Nachgang des vor einigen Jahren ausgerufenen pictorial turns und als Konsequenz der massenhaften Verbreitung sowie der Veralltäglichung des Gebrauchs fotografischer Technologie, kann die interpretative Soziologie heute auf ein Verfahren zurückgreifen, welches bei der Rekonstruktion vergangener Ereignisse neue Perspektiven eröffnet.

Im Gegensatz zur US-amerikanischen Forschung ist der Einsatz fotografischen Materials in der europäischen Soziologie kaum etabliert. Offenbar bedarf es noch weiterer Argumente, um diese ,Methode' in Europa hoffähig zu machen. Auch wenn es sich um eine betagte Technik handelt, muss sie derzeit noch hinsichtlich des Methodenkanons als neu oder ,innovativ' betrachtet werden. Im Folgenden wird die methodische ,Innovation` zunächst im Hinblick auf die Chancen eingeführt, die sich aus einer Verwendung fotografischen Materials in der sozialwissenschaftlichen Forschung ergeben. Dies kann im Hinblick auf das Problem rekonstruktiver Erforschung von vergangenen Ereignissen zugespitzt werden. Hierfür vermag der Einsatz visuellen Materials, durch das sich Elemente des Vergangenen ,konservieren' lassen, neue Impulse zu setzen. Die Integration von Bildern in die qualitative Forschung bedarf in diesem Zusammenhang keiner neuen Vorgehensweise - als Erzählstimulus können Fotografien weitgehend umstandslos in die vorliegenden Befragungsverfahren eingebettet werden. Im Mittelpunkt der Ausführungen steht eine Typisierung unterschiedlicher Möglichkeiten einer solchen Indienstnahme, wobei die Form des Interviews über eine initiierte Fotodokumentation (foto- 
elicitation) als besonders ,neu' herausgegriffen und einer näheren Betrachtung unterzogen wird. Anhand eines Anwendungsbeispiels aus der Forschungspraxis werden einzelne Momente des Verfahrens illustriert. Die Überlegungen schließen mit einem Resümee und einem Ausblick über Potenziale und Grenzen der Verwendung visueller Stimuli in der qualitativen Forschung.

\section{Das Bild als Text? Der Text zum Bild}

Nicht erst seit dem Ausruf eines so genannten pictorial turn (Mitchell 2008: 101ff) befasst sich die Soziologie intensiv mit der Analyse fotografischen Bildmaterials. ${ }^{1}$ Bemerkenswert ist, dass es eines turns bedurfte, um sich der Nutzung dieser Form empirisch verwertbarer Manifestationen bewusst zu werden. Bis dahin hatte die soziologische Arbeit mit Fotografien eher in den Grenzbereichen zur Anthropologie, Ethnografie, Volkskunde oder Kunstgeschichte stattgefunden und war - aus welchen Gründen auch immer ${ }^{2}$ - nicht salonfähig. Die Qualifizierung als turn im Sinne einer Neuausrichtung legitimer Orientierungen für empirische Forschung brachte einen Anerkennungszuwachs für diejenigen, die sich schon länger mit Bildern befasst hatten, ist sie doch Ausdruck einer späten Hinwendung , der ${ }^{6}$ Wissenschaft zum Bildmaterial.

Neben der Funktion des Fotografierens im Kunstschaffen, dient Fotografie in erster Linie der ,Aufbewahrung' des Vergänglichen. Für die soziologische Reflexion ist, lässt man das kunstsoziologische Erkenntnisinteresse beiseite, vor allem der ,konservierende' Gebrauch der Fotografie relevant. Ein Teil der Frage, warum ein Ereignis fotografisch festgehalten wurde, scheint damit beantwortet zu sein: weil es einmalig stattgefunden hat und in seiner Einmaligkeit für eine spätere Erinnerung bereitgehalten werden sollte (vgl. Harper 1988). Der andere Teil der Frage muss sich dann darauf richten, warum aus der Reihe dauernder - und das heißt: werdender und vergehender - Ereignisse eine Selektion getroffen wird. Im Gegensatz zur Wahrnehmung ist die fotografische Handlung dadurch sinnhaft, dass sie Erinnerbarkeit reflektiert vorbereitet.

Die fotografische Abbildung ist als Ergebnis dieser Handlung ein Zeichensystem, dessen Sinnschichten unabhängig von der Intention seiner Erzeugung analysiert werden können. Das Abgebildete ist oberflächlich im Rahmen eines kulturspezifischen Konsenses auszudeuten, wobei intersubjektiv davon ausgegangen wird, dass die Darstellung einen vergangenen Moment authentisch widerspiegelt. Bei genauerer Betrachtung werden die Bedeutungen gemäß des je subjektiven Erfahrungszusammenhangs und der daraus resultierenden Spezifika individueller Wahrnehmung differieren. Im Grunde ist es jedoch möglich, sich über den Inhalt einer Fotografie zu verständigen. ${ }^{3}$

Während die Manifestation visueller Informationen auf dem Bild bis zu einem gewissen Punkt unstrittig zu sein scheint, ist - als weitere Dimension - die Frage nach der subjektiven Sinnzumessung höchst problematisch. So könnte davon ausgegangen werden, dass je artifizieller eine Fotografie ist, ihre Deutbarkeit wiederum an kulturspezifische Codes und an man-

1 Ein genuin soziologischer Referenztext ist Siegfried Kracauers Theorie des Films, in der er sich ausführlich mit Fotografie befasst. Einflussreich für fotosoziologische Arbeiten sind auch Roland Barthes Studie Die helle Kammer sowie die Untersuchung von Gisèle Freund über Photographie und Gesellschaft.

2 Pierre Bourdieu et al. (2006) haben sich mit den Anerkennungsproblemen der Fotografie im ,Konzert ${ }^{\star}$ der Kulturtechniken beschäftigt. Auch die Soziologie ist nicht immun gegen kulturspezifische Wertmaßstäbe, womit sich die lange Zurückhaltung der Forschung gegenüber einer Indienstnahme dieses Mediums erklären könnte.

3 All dies sollte nur unter Berücksichtigung der vielschichtigen Analysen des Verstehens nach Alfred Schütz (1974) vorgenommen werden. 
nigfaltige Voraussetzungen im Hinblick auf die Interpretationskompetenz ihres Betrachters geknüpft ist. $^{4}$

Ein maßgebliches Kriterium ist hierbei die Zeitlichkeit des Dargestellten. Sobald ein Bild das Prädikat ,zeitlos` erhält, verweist dies auf ein umfassendes System kulturspezifischen Wissens und auf die Fähigkeit zur Decodierung. Ein Foto ,aus der Zeit' erscheint als unmittelbar verständlich; es wird beispielsweise nicht allegorisch gedeutet, sondern ,realistisch", also vor dem Hintergrund dessen, was es ,zeigt ${ }^{6}$ - und zwar ,objektive ${ }^{6}$ Wirklichkeit. ${ }^{5}$ Wenn die Herstellungsintention eines Fotos in der Dokumentation eines flüchtigen Ereignisses besteht, ist die Frage nach der Selektivität des Motivs anders zu beantworten als im Kontext künstlerischer Fotografie, der es nicht (primär) um die Erzeugung von Erinnerungsstimuli geht. Massenfotografie ist Dokumentation, die dem Wunsch entspringt Bedeutsames zu bewahren. Es soll etwas über die Zeit - und über die Gefahr des Vergessens - hinweggerettet werden.

Zunächst und mit Blick auf den Gebrauch der Alltagsfotografie beschränkt sich die Erzeugung von Erinnerungsstimuli auf die Biografiekonstruktion des Fotografen. Erinnerungsstimuli werden zur ,Identitätsarbeit" verwendet - entsprechend orientiert sich ihre Herstellung an identitätsrelevanten Ereignissen. Die Alltäglichkeit des Festhaltens korrespondiert dabei der Außeralltäglichkeit sowie der Vergänglichkeit des Motivs: Passageereignisse und -riten, Urlaubsmomente, seltene Begegnungen oder Situationen, die vor dem Hintergrund ihrer Endlichkeit und Vergänglichkeit festgehalten werden. Auch die Motivauswahl dieser Privatfotografie folgt sozial ,gewachsenen' Selektionsmustern. Die Zuordnung eines Fotomotivs als ,Erinnerung' oder als ,Kunst` lässt sich aus der Nähe oder Ferne zur Institutionalisierung der Motivauswahl ermessen. Künstlerische Fotografie sollte, mit anderen Worten, nicht einer institutionalisierten Motivik folgen, wie dies bei der selbstdokumentarischen Amateurfotografie - etwa bei den immer gleichen Urlaubsmotiven - festgestellt werden kann.

Aus soziologischer Sicht bieten nicht nur die fotografischen Manifestationen von Ereignissen eine Vielzahl von Deutungsmöglichkeiten. Die Bedeutungsgenese, die im Zuge des Erinnerns von Individuen durchgeführt wird, muss als vielschichtig erkannt werden. $\mathrm{Zu}$ unterscheiden ist zwischen einer Erinnerung, die sich auf eigens zu diesem Zweck hergestellte Stimuli bezieht und einer Erinnerung, die auf Stimuli gerichtet ist, welche kollektive Bedeutungen im Sinne von Erinnerungserwartungen repräsentieren. Es liegt nahe, zwischen der sozialen Funktion des Aufbewahrens einzelner Elemente vergänglicher Ereignisse und der von ihrem Entstehungsprozess abgelösten Gegenständlichkeit zu unterscheiden. Dabei handelt es sich um eine Reifizierung eines Erfahrungsgegenstandes. Solange der Gebrauch des Fotos nicht von seiner Erzeugungsintention abgelöst ist, ist das Foto ein Erinnerungsstimulus. Sobald es von dieser Intention getrennt wird, indem es - etwa in Folge seiner technischen Reproduzierbarkeit - seinen Entstehungszusammenhang verlässt, wird es, reduziert auf die in ihm manifestierte Information als Kulturartefakt, in neuer und anderer Weise deutungsbedürftig.

\section{Befragungen und das Problem des Bezugs auf Vergangenheit}

Für die soziologische Arbeit sind Fotografien daher aus zweierlei Sicht relevant: Einerseits erscheinen sie als Informationsträger im Hinblick auf kollektiv relevante Erinnerungen. Sie dienen als Hinweise auf soziale Bezugsrahmen des kollektiven Gedächtnisses (vgl. Halbwachs

4 Ähnliches gilt für die kommerzielle Erzeugung von Fotografien - auch hier sind kulturspezifische Codes aus dem Bereich des Marketingwissens oder der Werbepsychologie heranzuziehen, wenn eine adäquate Deutung vorgenommen werden soll.

5 Der Realitätsanspruch der Fotografie lässt sich auch vor dem Hintergrund der Wortfamilie ,objektiv ${ }^{6}$ verstehen: So wird die auf einen Gegenstand gerichtete Linsenkombination des Fotoapparats bekanntlich als ,Objektiv“ bezeichnet - ein technisches Gerät, das in der Lage ist, Dinge zu ,objektivieren“ (vgl. hierzu auch Köstlin 1995). 
2006). Andererseits werden sie als Merkzeichen (vgl. Schütz / Luckmann 1979: 328ff) der individuellen Erinnerung geschaffen und für eine wiederkehrende Verwendung vorgehalten. Beide Fälle sind für die soziologische Forschung relevant, da sie konstitutive Elemente der Konstruktion subjektiver wie kollektiver Identität darstellen. ${ }^{6}$ Will man sich über die Konstitutionsbedingungen gesellschaftlicher Wirklichkeit informieren, kann der Weg über eine Erforschung des individuellen wie kollektiven Gebrauchs von Fotografie aufschlussreiche Einsichten gewähren. Dabei geht es weniger um den manifesten Vergangenheitsbezug des dokumentarischen Bildes, als vielmehr um die an diesem und nur durch dieses Medium sich vollziehende Konstruktion von Erinnerungen.

Wenn weite Teile der soziologischen Arbeit zu Fotografien sich mit bildwissenschaftlichen beziehungsweise bildhermeneutischen Analysen befasst haben, ${ }^{7}$ wurde ein großes Potenzial dieses Mediums nicht ausgeschöpft. Die Suche nach objektiven oder objektivierbaren Sinnstrukturen der Herstellungsbedingungen einer Fotografie, deren Produzent nicht gehört wird oder werden kann, zwingt die Interpreten zur Spekulation. Demgegenüber ist die Beobachtung der Deutungspraxis im Hinblick auf Fotografien eine vom Bildinhalt systematisch abzugrenzende Forschungsstrategie, die sich auf die interpretative Konstruktion gegenwärtig relevanten Deutungswissens konzentriert. Die (Re-)Konstruktion dieses Wissens muss nicht durch den oder die Forscher(in) vorgenommen werden, sondern erfolgt durch für die Forschungsfrage relevante Personen oder Gruppen.

Das Verfahren der so genannten foto-elicitation ${ }^{8}$ besteht in der Hervorlockung von Informationen anhand fotografischen Materials, ${ }^{9}$ welches von den , beforschten ' Personen zum Untersuchungszweck hergestellt wurde. Es bietet einen weiteren Vorteil im Vergleich mit anderen Interviewverfahren, die zur Anwendung kommen, wenn es um die Rekonstruktion vergangener Erlebnisse geht: Erzählinterviews wie das narrative Interview sind durch eine Zurückhaltung der interviewführenden Person, die nondirektiv vorgehen soll, gekennzeichnet. Eine solche Befragungsform ist auf die aus der Interaktionssituation des Interviews hervorgehende Erzählung angewiesen. Die Verwendung von Fotografien bietet einen anderen, thematisch enger umgrenzten und zugleich weniger , atmosphärisch-sensiblen`Zugang.

6 Weitergehende Überlegungen zur Konstitution individueller und kollektiver Identität stellt Bernhard Giesen (2001) an.

7 Im Blickpunkt steht hier die Interpretation des Bildes aus Sicht der oder des bildwissenschaftlich oder bildhermeneutisch geschulten Forscherin oder Forschers. Für einen bildwissenschaftlichen Zugang im Rahmen der dokumentarischen Methode hat Ralf Bohnsack (2009) ein Standardwerk vorgelegt. Hermeneutisch geprägte Analysen finden sich im Kontext der ,objektiven Hermeneutik' nach Ulrich Oevermann (vgl. z.B. Haupert 1994).

8 Der Begriff der foto-elicitation wird von Douglas Harper (2002) verwendet - in die deutschsprachige Diskussion ,importiert` ihn Anna Brake (2009), die auch die Eindeutschung ,Foto-Elizitation“ vornimmt. Der Begriff ,Elizitation“ wird hier übernommen, um diese Form der fotogestützten Befragung $\mathrm{zu}$ charakterisieren.

9 In der US-amerikanischen Forschung gibt es mit Blick auf fotogestützte Erhebungsverfahren eine Tradition auf die hier nur hingewiesen werden kann. Unterschieden wird dort im Rahmen von kulturellen ,Bestandsaufnahmen ' zwischen Makroanalysen, die sich für eine phänomenologisch-narrative Analyse von Bildinhalten interessieren, Mikroanalysen, die Fotografien in Momente ihrer kulturellen oder theoriebezogenen Bedeutung zerlegen und den microimage-Ansatz, bei dem die Beziehungen, Ausdrücke und Bewegungen von oder zwischen abgebildeten Personen oder Dingen analysiert werden (vgl. Heisley / Levy 1991). Vorreiter in der Diskussion um den Einsatz visuellen Materials in Deutschland ist Stefan Guschker (2002), der einen Überblick über fotogestützte Verfahren in Verbindung mit einer exemplarischen Studie gibt. 


\section{Methodologische Überlegungen zum fotogestützten Interview}

Geht man von einer als Dokumentationsimperativ für biografische Erlebnisse zu bezeichnenden sozial vermittelten Ordnung der privaten Fotografie aus, die sich in einem weiteren Schritt im Arrangement des Fotoalbums manifestiert, wird man auf die private Sammlung von Fotografien als Datengrundlage für bestimmte Forschungsfragen zurückgreifen können. Von Interesse ist nicht nur die bildhermeneutische Auswertung des auf den gesammelten Fotos Abgebildeten; aufschlussreich ist die Anordnung der Aufnahmen im Archivierungsmedium. Die Art und Weise der Archivierung gibt Hinweise auf die spezifischen Ordnungsstrukturen und Relevanzen, die einer tradierten Logik thematischer oder chronologischer Anordnung folgen und - im Falle elektronischer Archivierung - einer ebenfalls tradierten individualistischen Aufbereitung gemäß der eigenen Wiederbetrachtungserwartung entsprechen. In Zeiten der massenhaften Verbreitung der Digitalfotografie beginnt die Ordnung des Archivs bei einer chaotisch anmutenden Speicherung von Bilddateien auf einem Datenträger, reicht über die Rubrizierung des Materials unter thematische ,Ordner ' bis hin zu detaillierten und kommentierten Arrangements der Bilder im Album. Entsprechendes ist auch bei der nicht digitalen Knipserfotografie ${ }^{10}$ anzutreffen: Fotoabzüge können in Schachteln gesammelt werden, eine (Un-)Ordnung, die die Möglichkeit differenzierterer Archivierung offenhält. Sie können in Mappen oder Alben sortiert und in spezifischer Weise für Erinnerungshandlungen und Erinnerungsrituale verfügbar gehalten werden. Das Fotoalbum - gleichviel, ob es einer privaten oder vielleicht auch geschäftlichen Nutzung dienen soll - beinhaltet ein auf bestimmte Regeln der ,Lesbarkeit" hinweisendes Erinnerungsangebot. ${ }^{11}$

Für die auf vergangene Ereignisse und Ereignisketten rekurrierende sozialwissenschaftliche Rekonstruktionsarbeit ergeben sich zwei Zugriffsmöglichkeiten: Man kann sich am Archiv orientieren, indem man sowohl dessen Ordnung als auch seine Inhalte untersucht. Oder man rekonstruiert über die Kommentierung der oder des Dokumentierenden und Archivierenden die intentionsgeleitete Praxis der Organisation des eigenen Erinnerns - sowohl aus systematischer als auch in inhaltlicher Sicht. ${ }^{12}$

Die Präferenz vieler Sozialforscher(innen) für die Analyse von Bildinhalten ist möglicherweise auf die Bedeutung von Fotografien als authentische Zeugnisse der Wirklichkeit zurückzuführen. Schon früh bediente sich die ethnografische Forschung fotografischen Materials im Sinne von Existenznachweisen. Damit unterlag sie dem alltagspraktischen Vertrauen auf die Dokumentationsleistung des fotografischen Bildes. Dass auch Fotografien manipulierbar sind, war im juristischen Kontext bald erkannt worden, weshalb der Einsatz von Fotografien als Beweismittel in Gerichtsprozessen lange Zeit als problematisch galt. Dort, wo nicht mit Fälschung aufgrund von Interessenkonflikten zu rechnen war, konnte sich analog zum Alltags-

10 Den Begriff der Schnappschuss- oder Knipserfotografie entwickelt Timm Starl (1996) in Abgrenzung zur Kunst- oder Porträtfotografie.

11 Starl sieht eine explizite Verbindung zwischen Fotografie, Archivierung, Erinnerung und Identität: Die fotografische Aufnahme ,entstand im Hinblick auf ihre spätere Betrachtung, die der Blick des Photographen bereits vorwegnahm; er machte eine Aufzeichnung wie die Eintragung in ein Tagebuch. So konnte [...] die Vergangenheit nicht aus den Augen verloren werden. Die Photographien im Album, selbst entworfen, hergestellt, geordnet und verwahrt, dienten der Vergewisserung einer Spur, die zu ihr führte. Im Blick auf das Bild - im Moment des Erinnerns - erschien der in der Vergangenheit fixierte Punkt als Anfang einer Linie des Lebens, der über die Gegenwart hinausreichte: Das Erinnerte wurde zu Illusion, das erkannte Gewesene zum geträumten Zukünftigen. Die Bilder zeigten nicht nur Ausschnitte des Daseins von sich und den Seinen, sondern sie fungierten als Orientierungszeichen, die - zur Lebensgeschichte verknüpft - auf die eigene Identität wiesen“" (Starl 1996: 74).

12 Das Archiv wird als Sammlung von als erhaltenswert erachteten Artefakten verstanden. Im Anschluss an Michel Foucault muss das Augenmerk auf den Archivar gerichtet werden, der letztlich darüber befindet, was bewahrt und was verworfen und damit vergessen wird (vgl. hierzu Keller 2011). 
gebrauch das Heranziehen von Fotografien als beweiskräftige Dokumente von Ereignissen in einer flüchtigen Wirklichkeit etablieren.

Mit Bildern, die ,mehr als 1000 Worte` zu sagen vermögen, ist nicht nur das Problem einer gewissen Mehrdeutigkeit und daraus resultierend einer ambivalenten Lesbarkeit verbunden. Im Hinblick auf die fototechnische Vergegenständlichungspraxis muss die Auswahl der Motive als eigenständige Sinnschicht behandelt werden, die einem angemessenen Bildverstehen zuträglich sein kann. Aus dieser Einsicht lässt sich beim Einsatz der Fotografie in der qualitativinterpretativen Sozialforschung folgern, dass Fotos nicht nur als Analysegegenstand Verwendung finden können, sondern als Hilfsmittel bei der Rekonstruktion situativ-kontextspezifischer Perspektiven auf subjektiv relevante Ereignisse oder Gegebenheiten. So können Personen mit Fotoapparaten ausgestattet und gebeten werden, entlang einer spezifischen Aufgabenstellung, ihre' Welt-Sicht festzuhalten. Auf diese Weise erzeugte Selbstdokumentationen lassen sich einerseits bildverstehend auswerten. Andererseits dienen sie als Grundlage von Berichten der Dokumentar(inn)e(n) über die von ihnen erstellten Bildersequenzen. Die folgenden Überlegungen konzentrieren sich auf diesen zweiten Aspekt der sozialwissenschaftlichen Arbeit mit fotografischem Material. Es geht nicht ausschließlich um die Arbeit mit Abgebildetem oder dem Zeichensystem ,Bild', sondern um die Analyse von Praktiken der Abbildung und der Archivierung ihrer Resultate. Solche eröffnen durch die technischen Möglichkeiten der Fotografie und die mit ihr verbundenen in westlich-modernen Gesellschaften massenhaft verbreiteten Dokumentationsgewohnheiten einen eigenständigen Zugang zur retrospektiven Rekonstruktion gesellschaftlicher Wirklichkeit.

Legt man den Akzent auf das Archiv, in dem die in einem bestimmten Zeitraum gesammelten Fotografien aufbewahrt werden, erschließt sich neben der Ordnung dieses Archivs im Zuge der Befragung die Art und Weise des erinnernden Zugriffs auf seine Inhalte. Dabei stehen neben der Information über das Abgebildete auch der je erinnernde Umgang und die aus diesem erwachsende Konstruktion einer spezifischen Vergangenheit im Mittelpunkt. Hier geht es nicht nur um eine fotogestützte Sammlung lebenslaufbezogener Daten. Im Blick steht auch die Art und Weise der Biografiekonstruktion anhand eines Fotoalbums. Neben einem solchen Interesse können fotografische Bildsequenzen auch zur je gegenwärtigen Rekonstruktion von Elementen kollektiver Identität aus der Sicht Einzelner verwendet werden oder zur Er-Innerung ${ }^{13}$ der Bedeutung und des Bedeutungszusammenhangs jedweder Bilderkombination. Angestrebt wird eine in der Form des Interviews angeregte und angeleitete Reflexion über Fotografien, die der privaten Dokumentation ebenso entstammen können wie jedem anderen Archiv bis hin zu willkürlichen Bildfolgen, die in einer quasi-experimentellen Versuchsanordnung durch eine ,Versuchsperson' miteinander kombiniert werden sollen.

Liegt der Akzent auf der Dokumentation beziehungsweise der Praxis des Abbildens, sind zwei Formen zu unterscheiden: Auf der einen Seite kann sich die fotogestützte Befragung auf Fotomaterial beziehen, das im Kontext eines Herstellungsauftrags mit Blick auf einen für die Forschungsfrage relevanten Gegenstand erzeugt wurde. Ein solcher Auftrag kann beispielsweise darin bestehen, den eigenen Wohn- und Lebensraum oder den eigenen Arbeitsplatz fotografisch ,festzuhalten“ (vgl. hierzu Wuggenig 1991). Durch die subjektive Relevanzsetzung und Selektion der Motive wird eine spezifische Weltsicht rekonstruierbar. ${ }^{14}$ Auf der

13 Auf diese Lesart von ,Erinnern“ weist Schütz hin: „Das Innewerden des Erlebens im reinen Ablauf der Dauer wandelt sich in jeder Phase, von Augenblick zu Augenblick in erinnertes Soeben-Gewesen; die Erinnerung ist es, welche die Erlebnisse aus dem unumkehrbaren Dauerablauf heraushebt und so die Urimpression des ,Innewerdens“ in ,Er-innerung“ modifiziert.“ (Schütz 1974: 64).

14 Dasselbe gilt für die Befragung von Gruppen. Hier wird die Selektion zur interaktiv auszuhandelnden Entscheidung - man einigt sich darauf, was als ,repräsentativ' und thematisch relevant aufgenommen wird. Einen Überblick über entsprechende Forschungsansätze gibt Anna Brake (2009). 
anderen Seite kann der Dokumentationsauftrag auch im Hinblick auf Ereignisse oder Ereignisketten ergehen. Die Dokumentationssituation ist hier anders einzuschätzen, da der oder die Dokumentar(in) unter dem Druck der kommenden und vergehenden Ereignisse steht, zwischen Dokumentation (Beobachtung) und Teilnahme oszilliert und im Zweifelsfall zwischen diesen beiden Modi der Teilhabe entscheiden muss. ${ }^{15}$ Wenngleich sich die Vergabe von Dokumentationsaufträgen in Verbindung mit einer korrespondierenden Befragung gut eignet, um den subjektiven Blick auf Ereignissequenzen retrospektiv zu rekonstruieren - man könnte in diesem Zusammenhang von einer Variante des prozessbegleitenden Interviews (vgl. Dimbath 2012) sprechen - muss dem Problem der Selektivität im Modus des Dokumentierens als eingeschränkter Teilhabe Rechnung getragen werden. ${ }^{16}$ Wer fotografiert, tanzt nicht. Die Haltung der Fotografin oder des Fotografen ist die eines aus dem Kern des Geschehens zurückgetretenen Beobachters. Wenngleich es die Foto-Elizitation in höherem Maße ermöglicht, Sequenzen abgelaufener Ereignisse in Erinnerung zu rufen und dadurch eine genauere Rekonstruktion zu ermöglichen, bringt es die fehlende Involviertheit der oder des Dokumentierenden mit sich, dass eine andere Erlebnisqualität berichtet wird, als dies bei einer narrativen Befragung zu erwarten wäre. ${ }^{17}$

Sowohl im Fall einer zeitpunktbezogenen als auch einer verlaufsbezogenen Dokumentation kann es in der Befragungssituation dem oder der Interviewpartner(in) anheimgestellt werden, in welcher Reihenfolge über die Aufnahmen gesprochen wird. Dass die Dokumentation einer Ereigniskette eine chronologische Reflexion nahelegen würde, ist keine notwendige Bedingung, da der Befragte erlebnisbezogene Schwerpunkte setzen oder die Erlebnisreihe umgekehrt darstellen kann. Zusammenfassend lassen sich die Anwendungsgebiete des fotogestützten Interviews unterscheiden:

Die Systematik richtet sich einerseits nach der Herkunft oder dem Produktionszusammenhang des fotografischen Materials, welches als Erzählanreiz für die Befragung herangezogen wird. Dabei kann entweder auf bereits vorhandene, eingelagerte und damit archivierte Bilder oder bezüglich der Forschungsfrage eigens, in Auftrag gegebene' Aufnahmen zurückgegriffen werden (Elizitation). Andererseits bietet sich eine Unterscheidung der Zeitbezüge der Fotoverwendung an, indem man nach einer eher zeitpunkt- und einer eher verlaufsbezogenen Bildersammlung differenziert. Der Zeitpunktbezug zielt auf das, was man als ,Momentaufnahme“ bezeichnen kann. Hier geht es um Material, das um ein bestimmtes Thema beziehungsweise die Forschungsfrage arrangiert ist. Der Verlaufsbezug ist durch die Dokumentation eines Prozesses oder einer Ereigniskette ausgewiesen - hier wird eine zeitliche Anordnung der als Erzählstimulus verwendeten Bilder relevant, da die durch die Bildbesprechung erzeugte Narration die Konstruktion einer Verlaufslogik impliziert.

15 Diese sehr subjektspezifischen Entscheidungen und Selektionen werden dann zu einer Auswertungsdimension eigener Qualität, wenn mehrere Dokumentar(inn)e(n) auf dieselbe Ereigniskette referieren.

16 Als weitere Schwierigkeit tritt hinzu, dass auch zwischen der Selektivität der Beobachtung der und der Selektivität der Dokumentation zu unterscheiden ist. Die Situation bringt aus der Sicht der oder des mit einer Dokumentation Beauftragten mindestens zwei ineinander verschachtelte Entscheidungsprobleme mit sich: Zunächst muss man sich dazu entscheiden, ob man an einem Geschehen unmittelbar oder mittelbar - als Beobachter - teilhaben möchte und darüber hinaus steht die Entscheidung an, welche der ablaufenden Ereignisse man für dokumentationsrelevant erachtet und welche nicht.

17 Dieses Problem veranlasst Forscher(innen) dazu, selbst zu fotografieren, um den Befragten dann vorab arrangierte Bildsequenzen - auf denen diese selbst zu sehen sind - vorzulegen (vgl. z.B. Heisley / Levy 1991). Hier ist mit anderen Ergebnissen zu rechnen, da für den Interviewpartner die Notwendigkeit entsteht, sich selbst zu objektivieren - es geht um die Kommentierung einer Fremdbeobachtung und nicht die des eigenen Beobachtens. 
Entlang dieser Dimensionen ergeben sich vier typische Verwendungen für die fotogestützte Befragung. Der Rückgriff auf archiviertes Material in der Kombination mit einer zeitpunktbezogenen Rekonstruktion vergangener Ereignisse im Sinne einer durch Forschungsinteressen angeleiteten Erinnerung ähnelt dem Zugang einer „Archäologie“ (Foucault 1988) der jüngeren Vergangenheit beziehungsweise einer Geschichtsschreibung, von unten'. Im Unterschied dazu lässt sich archiviertes Bildmaterial für die Analyse vergangener Abläufe nutzen. An dieser Stelle wird es zu einem wertvollen Stimulus des biografischen Interviews im Kontext der sozialwissenschaftlichen Biografieforschung. Sowohl aus ethnografischen Studien als auch aus der Lebensstilforschung ist der thematisch auf einen festgelegten Zeitpunkt orientierte Dokumentationsauftrag bekannt, dessen Resultate später zum Gegenstand der Befragung werden (vgl. Wuggenig 1991). Relativ neu erscheint gegenüber diesen drei Formen der Verwendung fotografischen Materials die Vergabe von Dokumentationsaufträgen an Personen, die in Ereignisketten oder Interaktionsverläufe eingebunden sind. Eine solche Ereignisverlaufsforschung muss als eine spezifische Beobachtungsform verstanden werden, bei der die Dokumentar(inn)e(n) - etwa im Gegensatz zur teilnehmenden Beobachtung - von Anfang an am Geschehen teilhaben. In ihrem intensiven und nicht durch die professionelle Distanz des Forschens behinderten Miterleben werden sie bestenfalls durch ihren Dokumentationsauftrag ,gestört'.

Abbildung 1: Anwendungshorizonte fotogestützter Interviews

\begin{tabular}{l|l|c|c}
\multicolumn{2}{c|}{} & \multicolumn{2}{c}{ Zeitbezug der Rekonstruktion } \\
\cline { 3 - 4 } \multicolumn{2}{c}{} & zeitpunktbezogen & verlaufsbezogen \\
\hline $\begin{array}{l}\text { Herkunft des } \\
\text { Fotomaterials }\end{array}$ & Archiv & „Archäologie“ & Biografieforschung \\
\cline { 2 - 4 } & Elizitation & Lebensstilforschung & Ereignisverlaufsforschung \\
\hline
\end{tabular}

Quelle: eigene Darstellung

Die in der hier skizzierten Systematik vorgestellten Verwendungsmöglichkeiten des fotogestützten Interviews eröffnen zwei Perspektiven. Beim Rückgriff auf Archivmaterial bieten sie eine andere Qualität der Evokation von Erinnerungen als dies bei Zeitzeugenbefragungen im Sinne reiner Narrativinterviews der Fall ist. Die Verwendung privater Archive bietet die Möglichkeit, nicht nur die im Bild enthaltene Information zu erfassen, sondern auch die biografischen Assoziationen, die für die Erzählenden , aus dem Bild‘ hinausweisen. Die beiden Varianten der Elizitation zielen auf die Erzeugung von Erinnerungen. Hier wird nicht explizit auf eine Vergangenheitsrekonstruktion gesetzt, sondern entweder auf eine ausgedehnte Gegenwart - die Wohnumgebung, der Arbeitsplatz, wie man sie ,dieser Tage“ erfährt - oder auf eine vergangene Erlebnissequenz. Der durch die visuellen Stimuli geschaffene Mehrwert besteht in der Möglichkeit Informationen zu generieren, die weit über das Abgebildete hinausgehen. Inwieweit die hierbei verfügbaren Informationen über die erzählbare Erfahrung von zurückliegenden Ereignissen hinausgehen, wurde bisher kaum erforscht. ${ }^{18}$ Dass die durch die Bildbetrachtung flankierte Erzählung jedoch eine andere Struktur aufweist und anderen Regeln der Konsistenzerzeugung folgt, ist anzunehmen.

\section{Durchführung und Auswertung elizitativer Interviews}

Der Stil der Interviewführung kann sich beim fotogestützten Interview an etablierten Formen des qualitativen Interviews ebenso orientieren wie abgrenzend profilieren. Weniger geeignet

18 Geradezu ,klassisch“ sind in diesem Zusammenhang die von John Collier (1957) angestellten Vergleiche zwischen unterschiedlichen Erzählstimuli im Kontext fotogestützter und nicht fotogestützter Befragung. 
erscheint zunächst das eine asymmetrische Befragungssituation nahelegende Erzähl- oder Narrativinterview wie es von Fritz Schütze (1983) für die Biografieforschung vorgeschlagen wurde. Da bei dieser Art der Befragung der Erzählstimulus in erster Linie vermittels der Generierung eines Erzählzwangs durch das Schweigen des Interviewers gesetzt wird und auf die aus dem Befragten ,hervordringenden " Narrationen in ihrer ,natürlichen " - und das meint: sich aus der Situation ergebenden - Abfolge Wert gelegt wird, steht hier eine situationsvermittelte Sequenz von stark aufeinander bezogenen Erinnerungsakten im Mittelpunkt. Die Grundstruktur des von Andreas Witzel $(1985 ; 2000)$ entwickelten problemzentrierten Interviews akzentuiert mit einem problemorientierten und zugleich für freie Narrationen geöffneten Leitfaden weniger einen singulären Erzählstrang als vielmehr eine immer wieder neu ansetzende und einem thematisch zentrierten Gespräch ähnelnde auf mehrere Erzählstimuli gestützte Informationsübermittlung. Aber auch die Struktur des problemzentrierten Interviews, bei dem mit einem offenen Erzählstimulus begonnen wird, dann einige auf das Forschungsinteresse bezogene Fragen nachgelegt und schließlich allgemeine Informationen per Kurzfragebogen eingeholt werden, ist durch eine vergleichsweise impulsarme Befragungsstrategie ausgewiesen. Eine besser geeignete Vorlage gibt das von Carsten G. Ullrich (1999) vorgestellte so genannte diskursive Interview ab, das einen aktiv beteiligten und kontroverse Stimuli setzenden Interviewer vorsieht. Die Fotosequenz, die dem Befragten durch den Interviewer vorgelegt und kommentierend mit ihm durchgegangen wird, ist Grundlage eines interessierten Gesprächs. Bei jedem Zugriff auf ein neues Foto beginnt eine Erzählsequenz mit einem durch das Bild repräsentierten Stimulus, wobei im Unterschied zum problemzentrierten Vorgehen die kommentierende Darstellung durch den oder die Dokumentar(in) sowohl im Hinblick auf den Bildinhalt als auch auf die Motivauswahl dialogisch-kritisch aber zurückhaltend hinterfragt werden kann. Das fotogestützte Interview beginnt mit einer kurzen Einleitung durch den oder die Interviewer(in), auf die ein offener Erzählstimulus folgt. Im Anschluss daran steht die Kommentierung von Fotografien im Mittelpunkt, wobei sich der oder die Interviewer(in) zunächst aktiv-zuhörend verhält - in der amerikanischen Diskussion werden diese Befragungsformen auch als autodriving bezeichnet (vgl. Heisley / Levy 1991), weil sich der oder die Befragte am visuellen Material orientiert und selbständig seine beziehungsweise ihre Erzählung entfaltet. Am Ende der Bilderdurchsicht nimmt der oder die Interviewer(in) die Möglichkeit wahr und stellt einzelne Fragen, die er/sie infolge einer Vorinterpretation der Bildersequenz - sofern eine solche vorgenommen werden konnte - entwickelt hat. Es empfiehlt sich nicht, die eigenen Bilddeutungen bereits während der Besprechung der Bilderfolge einzubringen, da der oder die Dokumentar(in) beziehungsweise Archivar(in) durch möglicherweise zu dominant vorgebrachte Standpunkte in der Entfaltung der eigenen Erzählung behindert werden könnte.

Nach der Charakterisierung und methodischen Verortung des fotogestützten Interviews soll im Folgenden auf das elizitative Vorgehen bei der Ereignisverlaufsrekonstruktion eingegangen werden. $\mathrm{Zu}$ unterscheiden ist nach der zunächst bewusst in den Hintergrund gestellten Interpretation des visuellen Materials und der stärker akzentuierten Deutung der infolge der Bilderschau entstandenen Erzählungen.

Noch vor der Konfrontation der Fotodokumentar(inn)e(n) mit dem von ihnen erstellten oder gesammelten Bildmaterial bietet sich eine kurze Durchsicht und Vorinterpretation seitens der Forscher(innen) an. ${ }^{19}$ Hieraus erwächst nicht nur die Möglichkeit, sich nicht von der Erzählung überrumpeln zu lassen und gegebenenfalls Fragen vorzubereiten, die im Hinblick auf einzelne Bilder nach Beendigung Erzählflusses gestellt werden können. Es besteht auch die Chance,

19 Diese Vorabbearbeitung wird bei Heisley / Levy (1991) als Makroanalyse bezeichnet - hierbei geht es mitunter darum, eine common-sense-Deutung als Kontrastfolie für die spätere Fotokommentierung zu entwickeln. 
erste Kontrastierungen zwischen dem ,neutralen' Bildverständnis der Forscherin oder des Forschers und den ,involvierten' Bedeutungszumessungen der oder des kommentierenden Dokumentars beziehungsweise der Dokumentarin zu erzeugen. Teil dieser Vorabauswertung ist auch eine Würdigung der Motivwahl, der Anzahl der Bilder sowie, im Fall der Dokumentation einer Ereigniskette, ein Abgleich mit anderweitig verfügbaren Informationen über die ,objektivierbare 'Struktur der Ereignissequenz, wie sie durch Zeit-, Stunden- oder Ablaufpläne vorgegeben wird. Es geht in keinem Fall darum, eine umfängliche Bildhermeneutik oder Analyse der Ikonologie anzustrengen, sondern lediglich um eine Vorinterpretation, die dazu dient, das Interview und weitere Auswertungsschritte vorzubereiten.

Nach einer solchen vorgelagerten Analyse besteht die Möglichkeit, den Prozess der Bezugnahme auf das Bildmaterial während des Interviews zu interpretieren. Hierzu empfiehlt sich die audiovisuelle Aufzeichnung (overhead) per Videokamera, die dabei hilft, das Zeigen des Bildes und seiner Details mit der Tonaufzeichnung des Interviews zu synchronisieren. Daraus ergibt sich die Möglichkeit, Informationsverlust und Fehldeutungen, die der bildlosen Transkription erwachsen können zu vermeiden. Die Erschließung des audiovisuellen Materials bedarf keiner hohen videoanalytischen Auswertungsschärfe, da die Protokollierung auffälliger Momente - etwa des gestischen Hinweises auf Bilddetails - sowie die präzise Dokumentation des Wechsels von einem Foto zum nächsten ausreichen, um die Interpretation des verschrifteten Interviews abzusichern.

Die Auswertung der fotogestützten Interviews kann sich entlang der Verfahren orientieren, wie sie in der qualitativen Sozialforschung entwickelt und praktiziert werden. Nach einer auch die Fokussierung auf das das Interview strukturierende Bildmaterial erfassenden - Transkription der Ton- und Bildaufzeichnungen bietet sich zunächst eine paraphrasierende Zusammenfassung und Rekonstruktion der Fälle an, um einen Überblick über die Bandbreite der Erzählungen zu gewinnen und erste Fallhypothesen zu formulieren. Beziehen sich die einzelnen Interviews auf ein und dasselbe Ereignis, bietet eine vergleichende Analyse der Motivauswahl und Dokumentationsdichte wie -breite weiteren Aufschluss im Hinblick auf die Vorbereitung einer tiefergehenden Analyse des verschrifteten Materials.

Eine solche kann sich dann - je nach Forschungsinteresse und Interpretationspräferenz sowohl hermeneutischer (vgl. die Beiträge in Hitzler / Honer 1997) als auch kodierender Verfahren (z. B. Strauss 1998) bedienen. Dabei sollte nicht nur an der Oberfläche nach Begebenheiten und damit nach ereignisbezogenen Episoden oder Erlebnissegmenten gesucht werden. Zentral ist, welche Bedeutungen die Befragten dem Erlebten über ihre Fotoerzählung beimessen. Nicht aus dem Blick geraten darf, dass Informationen darüber gewonnen werden müssen, wie sich das Subjekt zum Stimulus selbst in Beziehung setzt. Eine vollständige Interpretation umfasst somit die Sinnschicht

1. des über die Fotos vermittelten und anhand ihrer berichteten Ereignisses. Worin das dokumentarisch erfasste Ereignis besteht, erwächst in erster Linie der Darstellung der oder des Interviewten und lässt sich nicht ,automatisch' aus der Bildbetrachtung erschließen. Eine bildhermeneutische Deutung erscheint hier als unangemessen. Diese Ebene lässt sich als Themenevokation bezeichnen.

2. der mit diesem Bericht verbundenen, aber über das auf dem Bild Sichtbare hinausgehenden Narrationen und Bedeutungszumessungen. Die Stärke des Bildes besteht darin, dass der oder die Dokumentar(in) nicht auf die Kommentierung des Abgebildeten verwiesen ist. Das Bild ist dazu geeignet, mehrere Episoden in Erinnerung zu rufen. So gibt es immer eine Geschichte zur Relevanz des Abgebildeten für den Dokumentationsauftrag, aber auch eine Reihe von Geschichten, die beispielsweise mit abgebildeten 
Personen verbunden werden können. Diese Ebene kann als Kontextevokation bezeichnet werden.

3. der Reflexion über das Dokumentieren selbst. Mit der Beobachterrolle sind spezifische Momente verbunden, die entweder im Interview thematisch werden oder aus der Erzählung hervorgehen. So kann der Beobachter seine Distanziertheit bedauern oder begrüßen - entsprechend gestalten sich neben der Motivwahl auch die affektuelle Haltung zur aufgezeichneten Situation oder ihre Bewertung. Diese Ebene sollte daher als Situierungsevokation bezeichnet werden.

Aus der Erschließung der wechselseitig aufeinander verwiesenen Sinndimensionen lassen sich im Zuge abduktiver Interpretationsarbeit Deutungsmuster über abgelaufene Ereignisketten ermitteln. Über das Auffinden unterschiedlicher Sichtweisen auf ein singuläres Rahmenereignis werden durch Fallkontrastierungen typisierbare Erlebnis- und Sinndimensionen unterschieden, mit deren Hilfe sich die Qualität von Ereignissen als kollektive Erfahrung ermessen lässt. Der zu erwartende Erkenntnisgewinn bietet sich zur Analyse von Ereignissen an, deren komemorative Reflexion dadurch behindert ist, dass die Teilnehmenden keine Gelegenheit haben, durch gemeinsames Erinnern zu einer kollektiven Konstruktion dieses zeitlich begrenzten Wirklichkeitsausschnitts zu gelangen. Aber selbst im Fall narrativ reflektierter Kollektiverfahrungen vermag es die fotogestützte Befragung, die kommunikative Wirklichkeitskonstruktion zu unterlaufen, indem dem Erleben des Einzelnen in höherem Maße Rechnung getragen wird. Mit anderen Worten werden durch die Unterstützung einer retrospektiven Befragung durch einen letztlich subjektperspektivisch erzeugten Erinnerungsstimulus realistischere, weil weniger elaborierte Einzelwahrnehmungen und individuelle Relevanzen zur Geltung gebracht, die beispielsweise eine problemzentrierte oder durch themenspezifische Reflexionserwartungen flankierte, diskursive" Befragung systematisch ausblenden. Mit einer solchen ist das Problem verbunden, dass sie eine spezifische Reflexionspraxis beziehungsweise einen an der qualitativen Forschungspraxis orientierten und durch diesen strukturierten Narrationstyp erzeugen. Der maßgebliche Ertrag aus der Auswertung fotogestützter Interviews besteht darin, dass mit Blick auf die Themen-, Kontext- und Situationsdimension die Chance einer stärker auf vergangene Ereignisse rekurrierende Qualität von Informationen generiert wird.

\section{Ein Anwendungsbeispiel aus der Jugendreiseforschung}

Ein Beispiel für den gewinnbringenden Einsatz fotogestützter Interviews ist die Begleitforschung im Kontext von Veranstaltungen, die auf eine erzieherische oder sozialisatorische Wirkung zielen, aber aufgrund spezifischer institutioneller Regeln oder Vorgaben die Herausbildung von Erinnerungsgemeinschaften ausschließen. Nicht nur der durch Evaluationsanforderungen wachsende Legitimationsdruck auf Maßnahmen der Jugendhilfe, sondern die Bemühungen ihrer Anbieter um Selbstvergewisserung im Hinblick auf mögliche Wirkungen legt die Anwendung von Forschungsmethoden nahe, die über einfache paper-\&-pencil-Befragungen ebenso hinausgehen wie über Retrospektivinterviews mit weniger eloquenten und den Reflexionserwartungen von Forscher(inne)n mitunter nicht entsprechenden Kindern und Jugendlichen. Im Folgenden soll an einem Forschungsbeispiel auf wenige Aspekte der Verwendung eines fotogestützten Interviews zur Ereignisverlaufsrekonstruktion eingegangen werden. Der Hinweis auf einige Probleme aus der Praxis dieses Forschungszugangs steht hierbei im Mittelpunkt.

Ziel der hier vorgestellten wissenschaftlichen Begleitung einer Jugendreisemaßnahme im Kontext sogenannter Kurzzeitpädagogik war die Rekonstruktion der Sicht von 14-jährigen Jugendlichen auf die in diesem Zusammenhang gebotenen Erlebnischancen (vgl. Dimbath / 
Ernst / Holzinger / Wankerl 2008). Charakteristisch für diese Art von Veranstaltungen ist, dass sie eine Gruppe einander nicht oder kaum bekannter Personen zusammenführt. Diese verlassen ihre gewohnte Umgebung und verbringen eine begrenzte Zeit unter außeralltäglichen Bedingungen miteinander. Nach dem Ende des erlebnisintensiven und anstrengenden Zusammenseins wird die inszenierte Gemeinschaft wieder aufgelöst. Mit anderen Worten verlassen die Teilnehmenden einen ihre Alltagserfahrung und Alltagsroutinen scharf kontrastierenden Erlebniskontext, was aufgrund ausbleibender Erinnerungsstimuli und thematischer Relevanzstrukturen zu raschem Vergessen der hier gebotenen Erlebnisse und damit zu einer geringen Verankerung im subjektiven Erfahrungszusammenhang führen kann. Während für die Teilnehmenden das Erlebnis dieser Form organisierter Außeralltäglichkeit mit der als wohltuend empfundenen Dekontextualisierungswirkung eines Urlaubserlebnisses assoziiert wird, können sich verbandliche und nicht-kommerzielle Anbieter von Jugendreisen damit nicht zufrieden geben. Sie verfolgen durch Jugendreisemaßnahmen weitere Ziele, die zum einen in einem spezifischen Bildungsauftrag gemäß der vom Anbieter repräsentierten ,Ideologie‘ und zum anderen in der Hoffnung auf die Rekrutierung neuer Mitglieder bestehen. Aus ihrer Sicht kann sich eine wissenschaftliche Begleitung nicht auf Akzeptanzforschung beschränken; sie sollte auch Informationen über Wirkungen bereitstellen, die über konsumtive Erlebnisse hinausgehen.

Hier bietet sich die Verwendung fotogestützter Methoden an. ${ }^{20} \mathrm{Zu}$ Beginn der Veranstaltung erhält eine kleine Gruppe freiwilliger Fotodokumentar(inn)e(n) die Anweisung, alles zu fotografieren, was ihnen im Kontext ihres Reiseerlebnisses als ,wichtig' und ,festhaltenswert ${ }^{\text {‘ }}$ erscheint. ${ }^{21}$ Hierfür steht ihnen Material (Fotoapparate) in unbegrenztem Umfang zur Verfügung. Der Dokumentationsauftrag setzt die Bereitschaft voraus, sich eine Woche nach der Heimkehr zu den aufgenommenen Bildern befragen zu lassen.

Die auf diese Weise erzeugten visuellen Erzählstimuli erweisen sich als hochgradig heterogen in mehrerlei Hinsicht. So zeigte sich, dass die beteiligten Jugendlichen ihrer Aufgabe in sehr unterschiedlichem Umfang nachgekommen sind, indem sie in drei Tagen von 15 bis weit über 300 Bilder erzeugten. Bereits die Motivwahl, deren Heterogenität eher im Vergleich der Bildsequenzen als in der Vielfalt unterschiedlicher Motive innerhalb einer Sequenz aufscheint, eröffnet vielfältige Deutungsperspektiven. Bei allen Dokumentationen fällt auf, dass dem Fotoauftrag dann nachgegangen wird, wenn der oder die Dokumentar(in) nicht unmittelbar am Gruppengeschehen beteiligt ist - wenn er oder sie sich also eine Auszeit nimmt oder gerade keine Angebote stattfinden, die ein persönliches Engagement erfordern. In Verbindung mit den bildgestützten Erzählungen erweist sich, dass das individuelle Erleben der Maßnahme von den Erwartungen der Organisatoren abweicht. Die Aufnahmen zeigen neben erwartungsgemäß festgehaltenen Programmelementen (Themenevokation) vor allem andere Jugendliche: neu gewonnene Freunde aber auch solche, mit denen man nicht viel zu tun hatte oder haben wollte. $\mathrm{Zu}$ vielen abgebildeten Personen und Situationen werden Episoden angeboten, aus denen sich entnehmen lässt, wie die Befragungsteilnehmer ihre Interaktionen mit anderen ausgestaltet haben (Kontextevokation). Überraschend sind auch Motivarrangements, die überwiegend Na-

20 Aufgrund der sehr unterschiedlichen Beredsamkeit der Jugendlichen, die auch unterschiedliche Bildungshintergründe aufweisen, erscheint eine narrative oder problemzentrierte Retrospektivbefragung als ungeeignet. Die in solchen Zusammenhängen denkbare teilnehmende Beobachtung wird erstens ausgeschlossen, da die Anwesenheit von Beobachtern störend auf den Ablauf der Veranstaltung gewirkt hätte. Zweitens geht es um Erlebnisse und Erfahrungen und damit um subjektive Sinnzumessungen der Teilnehmenden, die durch Beobachtungsverfahren nicht gut erfasst werden können.

21 Das Forschungsinteresse richtete sich auf eine erlebnisnahe Rekonstruktion der Perspektive von Teilnehmenden - ohne weitergehende Anweisungen durch die Forscher(in). In der Literatur zur visuellen Soziologie finden sich auch Ansätze, die mit detaillierten Dokumentationsleitfäden, sogenannten shooting scripts, arbeiten (vgl. Suchar 1997). 
turbeobachtungen enthalten. Erzählungen hierzu zeigen, dass die Wahl eines idyllischen Veranstaltungsortes eine Erlebnisqualität eigener Art erzeugt hat, die von Personen wahrgenommen wurde, welche zur Reisegemeinschaft ebenso wie zum Programm der Veranstalter auf Distanz geblieben sind (Situierungsevokation).

Die starke Betonung von Erlebnissen, die zwischen den organisierten Programmeinheiten lagen, führte zur Entwicklung einer Hypothese über unterschiedliche Zeitregimes im Rahmen der Veranstaltung. Im Kontrast zu den Angeboten der Veranstalter fokussierten die Teilnehmenden auf die Zeitfenster, in denen sie sich ohne Programmvorgaben begegnen konnten. Die wichtigsten Erlebnisse waren nicht die ambitionierten, aufwändig inszenierten und ,ideologisch" wohl durchdachten Programmpunkte, sondern die Pausen, in denen sich die Jugendlichen unangeleitet begegnen konnten. Dies legt aber nicht den Schluss nahe, dass das organisierte Programm überflüssig wäre. Im Gegenteil schafft es durch seine Erlebnisdichte die Voraussetzung für ein Gemeinschafts- respektive Naturerleben in den Zeitintervallen, die den Jugendlichen zur freien Gestaltung überlassen bleiben. Eine explizite Deutung in dieser Richtung ist den Teilnehmenden kaum abzugewinnen. Sie bewerten - in einem an alle Jugendlichen am Ende der Veranstaltung verteilten Fragebogen - fast alle organisierten Programmpunkte als überaus gelungen. Die Reflexion über und Kommentierung von pubertären Selbstpräsentationen sowohl der Knaben als auch der Mädchen am Volleyballfeld in der Mittagspause, über den Krach mit den Freundinnen, das lange Suchen und Finden eines Gleichgesinnten oder die Kommentierung szenespezifischer Bekleidung anderer Jugendlicher sind in ihrer Vielfalt in anderen qualitativen Datenerhebungssettings möglicherweise nicht in diesem Umfang zu erwarten. Aufschlussreich für das Selbstvergewisserungsinteresse des Veranstalters waren neben diesen eher ethnografischen Informationen vor allem die Befunde zu unterschiedlichen Zeitwahrnehmungen beziehungsweise zur unterschiedlichen Bedeutungszumessung innerhalb des vorgegebenen Zeitregimes sowie eine infolge der Interviewauswertungen erstellte Typik unterschiedlicher Integrationsmodi im Rahmen der Gegebenheiten kurzzeitpädagogischer Gruppenveranstaltungen, die dazu in der Lage ist, ,pathologische“ Verläufe und Arrangements systematisch zu identifizieren.

\section{Diskussion und Fazit}

In diesem Beitrag wurde eine Differenzierung unterschiedlicher Formen des fotogestützten Interviews vorgenommen. Die durch einen pictorial turn ausgerufene Akzentverschiebung der soziologischen Forschung auf visuelle Darstellungen wurde nicht in den Mittelpunkt gerückt. Die stärkere Einbeziehung des Bildhaften in die soziologische Analytik kann zu einem Akzeptanzgewinn der mittelbaren Verwendung von Bildmaterial in der qualitativen Forschung beitragen. Für die (Re-)Konstruktion des vergangenen Erlebens bieten die technischen Entwicklungen der Massenfotografie sowie die aus ihnen erwachsenden kulturellen Veränderungen neue Wege. Bemerkenswert ist dabei, dass die Nutzung privater Fotografie in der Sozialforschung kein Novum ist; aber offenbar führen zwei Entwicklungen erst in jüngerer Gegenwart zu einer Aufwertung dieses Zugangs: Da ist zunächst der heute in vielen Schichten und Milieus verbreitete und vor allem preisgünstige Gebrauch der Fototechnik im Rahmen veralltäglichter Biografiekonstruktion und Identitätsarbeit. Eng damit verbunden ist die hier als Dokumentationsimperativ ausgewiesene soziale Praxis, bestimmte Momente eines institutiona- 
lisierten Lebenslaufs ${ }^{22}$ mit Fotos - oder in noch jüngerer Zeit auch mit Filmen - in einer bestimmten semiotischen Form aufbewahren und für späteres Erinnern vorhalten zu wollen.

Während sich die Forschung diese Imperative im Kontext von oral history oder Biografieforschung im Rückgriff auf das Material aus der alltäglichen Privatarchivierung zunutze machen kann, nutzt die Foto-Elizitation den soziokulturell eingelebten Umgang mit Fototechnik, um die Bedeutung von Ereignisketten erlebnisnäher zu rekonstruieren, als dies mit den Verfahren qualitativ-sinnrekonstruktiver Forschung bisher möglich war. Entscheidend ist hier im Vergleich zu älteren Experimenten mit Fotodokumentationen, dass der alltägliche Gebrauch der Fotografie heute nichts Besonderes mehr ist: Die Heutigen ,knipsen', archivieren und erzählen über Fotos, ohne sich noch über diese unfassbare Technik zu wundern. Hinzu kommt, dass der Preis nicht mehr hoch und das umständliche Prozedere der Bildentwicklung für den Massenkonsum organisiert und unkompliziert geworden ist. (Digital-)Fotografie gehört zur Normalität und hat dadurch eine neue Relevanz auch für die Sozialforschung gewonnen.

Die Stärke der elizitativen Fotobefragung liegt, so lässt sich zusammenfassen, zunächst in der Komplexität des Bildes als Erzählstimulus. Das fotogestützte Interview regt zu Erzählungen an, die unterschiedliche Elemente eigenen Erlebens in Erinnerung rufen. Diese Erinnerungen weisen in der Regel ebenso über das Abgebildete hinaus, wie bestimmte Elemente des Bildes nicht adressierbar bleiben - was thematisiert wird, obliegt dem oder der befragten Dokumentar(in). Hinweise auf nicht berücksichtigte Bildelemente führen nicht, automatisch ' zur erwünschten Explikation, da die Bilderstellung auch eine Überschussproduktion ist. Eine weitere Stärke besteht in der Chance, Einblick in Vorgänge zu nehmen, zu denen der unkomplizierte Zugang für die Forschung verstellt ist. Dies betrifft nicht nur erlebnisintensive Bereiche wie das hier dargestellte Gebiet kurzzeitpädagogischer Maßnahmen, sondern kann sich auch auf Abläufe in Organisationen, Religionsgemeinschaften oder Events richten. Als methodischer Ertrag hervorzuheben bleibt erstens die Erschließung einer besonderen Qualität der Konstruktion individueller Retrospektiven und zweitens der alternative Zugang zu einer Rekonstruktion vergangener Ereignisse aus unterschiedlichen Perspektiven.

Probleme, die sich im Kontext der Foto-Elizitation ergeben, sind in erster Linie die nicht kontrollierbare Synchronisation von Dokumentationshandlung und subjektivem Erleben. Damit verbunden ist die Gefahr, dass ein Dokumentationsauftrag zur Produktion von Bildern führt, die weder mit dem ,objektiven' Ablauf der Ereignisse noch mit den Sinnzumessungsund Wahrnehmungsstrukturen des Subjekts übereinstimmen. Unter diesen Umständen gerät die fotogestützte Befragung zu einer Annäherung über Residualinformationen. Auch eine solche kann zu aufschlussreichen und anderweitig nicht erfassbaren Informationen führen - sie ist jedoch recht weit von der Erwartung entfernt, durch die Bilderschau zum intersubjektiv relevanten Kern vergangener Ereignisse vorzudringen. Chancen und zugleich Risiken birgt der Umstand, dass sich die elizitative Fotografie an kulturell eingelebten Dokumentationsimperativen sowie einer eingeübten Praxis der Motivselektion und Bildgestaltung orientiert. Fotografie ist, sofern sie nicht im Kontext von Fotokunst erzeugt wird, keine Entäußerungstechnik von Innerlichkeit. Inwieweit sich die fotografische, Wahrnehmung ${ }^{6}$ alltäglicher ebenso wie außeralltäglicher Ereignisse an eingelebten Formen orientiert, kann jedoch erst durch weitere Forschung auf diesem Gebiet ermessen werden. Für die Foto-Elizitation werden solche in nächster Zukunft zu generierenden Befunde zu Etappen ihrer method(olog)ischen Etablierung und Weiterentwicklung.

22 Auch wenn Martin Kohli seine These vom institutionalisierten Lebenslauf vor dem Hintergrund spätmoderner Umbrüche teilweise zurückgenommen hat, ist die Feststellung nach wie vor zutreffend, dass die Konstituierung von Lebensläufen viele institutionalisierte oder typische Elemente aufweist. Insofern mag es als gerechtfertigt erscheinen, an der Beobachtung einer gewissen Gleichförmigkeit und Typizität von Lebensläufen festzuhalten (vgl. Kohli 1985). 


\section{Literatur}

Barthes, R. (1989): Die helle Kammer. Bemerkung zur Photographie, Frankfurt / Main.

Bohnsack, R. (2009): Qualitative Bild- und Videointerpretation. Die dokumentarische Methode, Opladen.

Bourdieu, P. / L. Boltanski / R. Castel / J.-C. Chamboredon / G. Langeau / D. Schnapper (2006): Eine illegitime Kunst. Die sozialen Gebrauchsweisen der Photographie, Hamburg.

Brake, A. (2009): Photobasierte Befragung, in: S. Kühl / P. Strodtholz / A. Taffertshofer (Hrsg.), Handbuch Methoden der Organisationsforschung. Quantitative und Qualitative Methoden, Wiesbaden, S. 369-388.

Collier, J. (1957): Photography in Anthopology: A Report on Two Experiments, in: American Anthropologist 59, S. 843-859.

Dimbath, O. (2012): Rekonstruktion ,großer' Entscheidungen. Entscheidungsverlaufsanalyse mithilfe prozessbegleitender Interviews, in: sozialersinn 13, S. 305-322.

Dimbath, O. / M. Ernst / E. Holzinger / C. Wankerl (2008): Elemente einer Soziologie der Jugendfreizeit, in: Deutsche Jugend: Zeitschrift für die Jugendarbeit 56, S. 118-127.

Foucault, M. (1988): Archäologie des Wissens, Frankfurt / Main.

Freund, Gisèle (1974): Photographie und Gesellschaft, München.

Giesen, B. (2001): Voraussetzung und Konstruktion. Überlegungen zum Begriff der kollektiven Identität, in: C. Bohn / H. Willems (Hrsg.), Sinngeneratoren. Fremd- und Selbstthematisierungen in soziologischhistorischer Perspektive, Konstanz, S. 91-110.

Guschker, S. (2002): Bilderwelt und Lebenswirklichkeit. Eine soziologische Studie über die Rolle privater Fotos für die Sinnhaftigkeit des eigenen Lebens, Frankfurt / Main.

Halbwachs, M. (2006): Das Gedächtnis und seine sozialen Bedingungen, Frankfurt / Main.

Harper, D. (1988): Visual Sociology: Expanding Sociological Vision, in: The American Sociologist 19, S. 54-70.

Harper, D. (2002): Talking about Pictures: A Case for Photo Elicitation, in: Visual Studies 17, S. 13-26.

Haupert, B. (1994): Objektiv-hermeneutische Fotoanalyse am Beispiel von Soldatenfotos aus dem Zweiten Weltkrieg, in: D. Garz / K. Kraimer (Hrsg.), Die Welt als Text. Theorie, Kritik und Praxis der objektiven Hermeneutik, Frankfurt / Main, S. 281-314.

Heisley, D.D. / S.T. Levy (1991): Autodriving: A Photoelicitation Technique, in: Journal of Consumer Research 18, S. 257-272.

Hitzler, R. / A. Honer (Hrsg.) (1997): Sozialwissenschaftliche Hermeneutik, Opladen.

Keller, R. (2011): Der Archivar und das Vergessen: Michel Foucault, in: O. Dimbath / P. Wehling (Hrsg.), Soziologie des Vergessens. Theoretische Zugänge und empirische Forschungsfelder, Konstanz, S. 113-137.

Kohli, M. (1985): Die Institutionalisierung des Lebenslaufs. Historische Befunde und theoretische Argumente, in: KZfSS 37, S. 1-29.

Köstlin, K. (1995): Photographierte Erinnerung? Bemerkungen zur Erinnerung im Zeitalter ihrer technischen Reproduzierbarkeit, in: R. Schenda / U. Brunold-Bigler / H. Bausinger (Hrsg.), Hören Sagen Lesen Lernen: Bausteine zu einer Geschichte der kommunikativen Kultur, Bern, S. 395-410.

Kracauer, S. (1985): Theorie des Films. Die Errettung der äußeren Wirklichkeit, Frankfurt / Main.

Mitchell, W.J.T. (2008): Bildtheorie, Frankfurt / Main.

Schütz, A. (1974): Der sinnhafte Aufbau der sozialen Welt. Eine Einleitung in die verstehende Soziologie, Frankfurt / Main.

Schütz, A. / Luckmann, T. (1979): Strukturen der Lebenswelt, Band 1. Frankfurt / Main.

Schütze, F. (1983): Biographieforschung und narratives Interview, in: Neue Praxis 13, S. 283-293. 
Starl, T. (1996): Erinnern um zu vergessen. Zur Entstehung der Bildwelt der Knipser, in: A. Volk (Hrsg.), Vom Bild zum Text. Die Photographiebetrachtung als Quelle sozialwissenschaftlicher Erkenntnis, Zürich, S. 67-82.

Strauss, A.L. (1998): Grundlagen qualitativer Sozialforschung: Datenanalyse und Theoriebildung in der empirischen und soziologischen Forschung, München.

Suchar, C.S. (1997): Grounding Visual Socioloy Research In Shooting Scripts, in: Qualitative Sociology 20, S. 33-55.

Ullrich, C.G. (1999): Deutungsmusteranalyse und diskursives Interview, in: Zeitschrift für Soziologie 28, S. 429-447.

Witzel, A. (1985): Das problemzentrierte Interview, in: G. Jüttemann (Hrsg.), Qualitative Forschung in der Psychologie. Grundfragen, Verfahrensweisen, Anwendungsfelder, Weinheim, S. 227-255.

Witzel, A. (2000): Das problemzentrierte Interview, in: Forum Qualitative Sozialfoschung 1, abrufbar unter: http://qualitative-research.net/fqs, letztes Abrufdatum: 15.3.2013.

Wuggenig, U. (1991): Die Fotobefragung, in: H. Kreutz (Hrsg.), Pragmatische Soziologie. Beiträge zur Diagnose und praktischen Lösung gesellschaftlicher Gegenwartsprobleme, Opladen, S. 333-354.

PD Dr. Oliver Dimbath Universität Augsburg Lehrstuhl für Soziologie Alter Postweg 101 (BCM) 86149 Augsburg oliver.dimbath@phil.uni-augsburg.de 